\title{
Laboratory Test Reference Range Lower Limit Original Unit
}

National Cancer Institute

\section{Source}

National Cancer Institute. Laboratory Test Reference Range Lower Limit Original Unit. NCI Thesaurus. Code C83095.

The lower limit of the laboratory test reference range, as reported in original units. 